\title{
Empirical slip and viscosity model performance for microscale gas flow
}

\author{
Matthew J. McNenly ${ }^{1, *, \dagger}$, Michael A. Gallis ${ }^{2}$ and Iain D. Boyd ${ }^{1}$ \\ ${ }^{1}$ Department of Aerospace Engineering, University of Michigan, Ann Arbor, MI 48109, U.S.A.

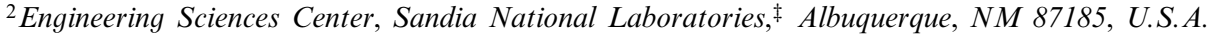

\begin{abstract}
SUMMARY
For the simple geometries of Couette and Poiseuille flows, the velocity profile maintains a similar shape from continuum to free molecular flow. Therefore, modifications to the fluid viscosity and slip boundary conditions can improve the continuum based Navier-Stokes solution in the non-continuum nonequilibrium regime. In this investigation, the optimal modifications are found by a linear least-squares fit of the Navier-Stokes solution to the non-equilibrium solution obtained using the direct simulation Monte Carlo (DSMC) method. Models are then constructed for the Knudsen number dependence of the viscosity correction and the slip model from a database of DSMC solutions for Couette and Poiseuille flows of argon and nitrogen gas, with Knudsen numbers ranging from 0.01 to 10 . Finally, the accuracy of the models is measured for non-equilibrium cases both in and outside the DSMC database. Flows outside the database include: combined Couette and Poiseuille flow, partial wall accommodation, helium gas, and non-zero convective acceleration. The models reproduce the velocity profiles in the DSMC database within an $L_{2}$ error norm of 3\% for Couette flows and 7\% for Poiseuille flows. However, the errors in the model predictions outside the database are up to five times larger. Copyright (c) 2005 John Wiley \& Sons, Ltd.
\end{abstract}

KEY WORDS: microscale gas flow; DSMC; slip models

\section{INTRODUCTION}

The lack of accurate and efficient simulation methods for micro-scale gas flows is directly due to two factors: the small length scales and slow bulk gas velocities associated with micro-scale devices. When the length scale of the flow approaches the mean free path of the

\footnotetext{
${ }^{*}$ Corrrespondence to: M. J. McNenly, 2012 FXB Building, 1320 Beal St., Ann Arbor, MI 48109, U.S.A.

†E-mail: monenly@engin.umich.edu

${ }^{\ddagger}$ Sandia is a multiprogram laboratory operated by Sandia Corporation, a Lockheed Martin Company, for the United States Department of Energy's National Nuclear Security Administration under contract DE-AC04-94AL85000.

Contract/grant sponsor: Department of Energy

Contract/grant sponsor: Sandia National Laboratories
}

Received 26 May 2004

Revised 26 May 2004

Copyright (c) 2005 John Wiley \& Sons, Ltd. Accepted 4 May 2005 
operating fluid, there is no longer a sufficient number of collisions between gas molecules to achieve local thermodynamic equilibrium (LTE). The degree that a gas flow deviates from LTE is typically measured by the Knudsen number $K n=\lambda / L$, where $\lambda$ is the mean free path between gas molecules and $L$ is the characteristic length of the fluid flow. As the Knudsen number increases, there are fewer collisions occurring within the length scale of interest, and the gas deviates further from LTE. A flow with a higher Knudsen number is said to be more rarefied because the number of molecules within the volume of interest is lower.

Non-continuum non-equilibrium regions cannot be accurately predicted using the continuum based Navier-Stokes equation, because near thermodynamic equilibrium is assumed for the noslip boundary condition and the transport closure. Particle simulations like the direct simulation Monte Carlo (DSMC) method of Bird [1], are correct in the non-equilibrium regions but suffer from statistical noise in the bulk velocity because of the random, or thermal, molecular motion. When the bulk velocity is much slower than the thermal velocity, many independent samples are needed to eliminate the statistical scatter and recover the bulk flow properties - as is typical for gas flows in micro-electro-mechanical systems (MEMS). In fact, for nitrogen gas at room temperature, the standard deviation in the molecular speed is about $300 \mathrm{~m} / \mathrm{s}$, which would require approximately 9 million independent samples in DSMC to reduce the scatter in the bulk velocity to $0.1 \mathrm{~m} / \mathrm{s}$. For MEMS gas flows that operate in the $\mathrm{mm} / \mathrm{s}$ range, the number of required samples can grow into the trillions; simulations for such conditions on the fastest supercomputers in the world can take weeks. DSMC is impractical in these cases as a tool to rapidly evaluate MEMS design iterations. Overall, the small length scales and slow bulk gas velocity combine to make continuum solutions inaccurate, and particle solutions time consuming.

Slip models have been proposed to improve the predictions of continuum methods for the non-equilibrium regions near solid boundaries. The idea is to relax the traditional no-slip boundary condition to allow the rarefied gas to slip at the wall. Slip models have been around since the beginning of gas kinetic theory, when Maxwell derived a relation between the slip velocity at the wall and the local velocity gradient [2-4]. For the models considered in this investigation, the slip model is the same form as Maxwell's original model with the addition of an empirical slip coefficient $C_{\mathrm{s}}(K n)$ that can be a function of the Knudsen number and is dependent on the flow type

$$
u_{\mathrm{s}}=\left.\frac{2-\sigma_{v}}{\sigma_{v}} C_{\mathrm{s}}(K n) \lambda \frac{\partial u}{\partial n}\right|_{w}=\left.\Lambda \frac{\partial u}{\partial \eta}\right|_{w}
$$

In the slip model equation (1), $u_{\mathrm{s}}$ is the slip velocity at the wall, $\sigma_{v}$ is the tangential momentum accommodation coefficient (TMAC), and $\Lambda=\left[\left(2-\sigma_{v}\right) / \sigma_{v}\right] K n C_{\mathrm{s}}(K n)$ is the non-dimensional form of the coefficient when the gradient at the wall $\partial u /\left.\partial n\right|_{w}$ is properly normalized. The TMAC characterizes the wall interaction for a colliding gas molecule as either a diffuse or specular reflection, and is defined as the ratio of diffuse reflections to the total number of reflections. In recent times, the desire for physically accurate and numerically efficient simulation of MEMS gas flows has renewed interest in the field and has brought to light a century of work. After Maxwell, many researchers contributed slip models based on extensions of higher order equations, including: Deissler, Cercignani and Kogan [5-7]. While more recent work by Karniadakis, Beskok and Pan has involved empirical models [8-11]. The above research represents only a fraction of the proposed models, yet almost all of them can be considered an extension of Maxwell's original model. 
While slip models have been shown to successfully predict gas flows near equilibrium, when the flow deviates far enough from equilibrium, the continuum solution is no longer physically valid and one cannot predict flows with a slip model correction alone [8]. As the flow deviates further from equilibrium, insufficient numbers of collisions throughout the flow cause the shear stress closure in the continuum solution to breakdown. Recently, Beskok and Karniadakis [8], and Bahukudumbi et al. [10] have proposed an empirical correction to the shear stress closure that allows the continuum solutions of simple flows to capture with high accuracy the non-equilibrium results. For simple cases, like Couette and Poiseuille flows, the velocity profile maintains a nearly similar shape from the continuum regime to the free molecular regime, which means that the continuum solution has a similar velocity profile to the free molecular flow solution. Using the slip model correction and shear stress correction as two free parameters to tune, the shape of the continuum solution can be stretched to fit any non-equilibrium solution for simple flows. The drawback is that one needs access to the nonequilibrium solutions in the first place in order to determine the best continuum corrections. There is no consensus in the literature about the limits of applicability of such corrections; however, the method should be useful for predicting non-equilibrium solutions of flows that are a small deviation from the known solutions.

This investigation will focus on evaluating empirical models for continuum corrections constructed from known non-equilibrium solutions. The known non-equilibrium solutions are found using the DSMC of Bird [1] to predict one-dimensional Couette and Poiseuille flows for argon and nitrogen gas flows with Knudsen numbers ranging from 0.01 to 10 . The best slip velocity and momentum transport corrections are determined by the continuum solutions that match the DSMC velocity and shear stress data in the linear least-squares sense for all flow conditions. In this investigation, our Couette and Poiseuille models each consist of two empirical models; one for the slip velocity correction and another for viscosity correction. Our Couette and Poiseuille models are constructed to capture the Knudsen number dependence of the slip and viscosity corrections for Knudsen numbers ranging from 0.01 to 10 . Each model is tested to determine how accurately it captures the non-equilibrium solutions in the database it was derived from, how sensitive it is to different flow types, and how it compares to the unified Couette model developed by Bahukudumbi et al. (BPB model) [10] and the unified Poiseuille model developed by Karniadakis and Beskok (KB model) [8]. Both the BPB and KB models use an empirical slip model for the boundary conditions and an empirical correction in the viscosity or shear stress for the transport closure. However, the functional form of the Knudsen number dependence is different in all cases. The BPB model uses a four-term, non-linear function to capture the Knudsen dependence of the slip model (2), while the transport closure uses a three-term polynomial ratio (3) that preserves the asymptotic behaviour of the shear stress in the continuum and free molecular limits.

$$
C_{\mathrm{s}}=a_{0}+a_{1} \tan ^{-1}\left(a_{2} K n^{a_{3}}\right)
$$

where $a_{0}=1.2977, a_{1}=0.71851, a_{2}=-1.17488$, and $a_{3}=0.58642$ are empirical coefficients.

$$
\pi_{x y}=-\frac{\beta K n^{2}+2 \gamma K n}{\beta K n^{2}+\delta K n+\gamma}
$$


where $\pi_{x y}$ is the shear stress normalized by the shear stress in the free molecular limit $\left(\tau_{x y}\right)_{\infty}$, and $\beta=0.5269, \gamma=0.6029$, and $\delta=1.6276$ are empirical coefficients.

$$
\left(\tau_{x y}\right)_{\infty}=\rho\left(U_{1}-U_{2}\right) \sqrt{\frac{2 k T_{\mathrm{w}}}{\pi m}}
$$

where $\rho$ is the fluid density, $U_{1}$ and $U_{2}$ are the lower and upper wall velocities, respectively, $k$ is the Boltzmann constant, $T_{\mathrm{w}}$ is the wall temperature, and $m$ is the mass of the gas molecule. The KB model has a functional form for the Knudsen dependence of the slip model that maintains high-order accuracy in the near continuum limit (5), and the corrected viscosity $\mu^{\prime}$ is based on a first principles analysis of the momentum transport in the continuum and free molecular limits (6).

$$
C_{\mathrm{s}}=\frac{1}{1-b_{0} K n}
$$

where $b_{0}$ is a generalized slip coefficient and $b_{0}=-1$ for Poiseuille flows, and

$$
\mu^{\prime}=\mu_{0} \frac{1}{1+\alpha K n}
$$

where $\mu_{0}$ is the fluid viscosity of the gas at a specified temperature, $\alpha$ is a rarefaction correction parameter that can be obtained from simulations or experiments. The functional form for the BPB slip model is used as the framework for our Couette and Poiseuille models because it is simple to set the asymptotic behaviour in the continuum limit and capture the transition in the empirical corrections for Knudsen numbers between 0.1 and 10 . The purpose of this investigation is not to propose better models than already found in the literature; rather, its goal is to gain insight in the construction of these unified, empirical models and the limits in their range of applicability. To this end, the Couette and Poiseuille models developed in this investigation are used to predict several different types of flow conditions not included in the non-equilibrium database, including: interpolation and extrapolation of Couette and Poiseuille flow conditions in the database, combination Couette and Poiseuille flow, partial wall accommodation, helium gas flows, and body force driven flow with uniform suction and injection.

In subsequent sections of this paper, the effects of non-equilibrium and the ability of slip models and shear stress closure correction to allow for continuum solutions to accurately capture non-equilibrium flows is demonstrated. The investigative methods are covered for: the DSMC simulation, the usage of the empirical corrections in the analytical continuum solutions, the least-squares approach for determining the best empirical corrections, and the construction of our Couette and Poiseuille models. The accuracy and sensitivity of the models compared to the solutions they were derived from is shown. Finally, the predictive power of our models is examined for flows outside the database used to construct the models.

\section{CORRECTION FOR NON-EQUILIBRIUM}

All gas flows, in equilibrium or not, must satisfy the conservation laws of mass, momentum, and energy. For continuum flows, these conservation laws are represented by a set of five 
differential equations, with additional transport closures for momentum and energy and a state equation, for which there is no known general solution. Assuming isothermal flow and the Newtonian shear stress closure, the conservation of energy is automatically satisfied, which results in a system of equations consisting of the continuity equation (7), the Navier-Stokes equation (8) and the shear stress closure (9).

$$
\begin{gathered}
\frac{\partial \rho}{\partial t}+\frac{\partial \rho v_{i}}{\partial x_{i}}=0 \\
\frac{\partial v_{i}}{\partial t}+v_{j} \frac{\partial v_{i}}{\partial x_{j}}=\frac{1}{\rho}\left(\frac{\partial \tau_{i j}}{\partial x_{j}}-\frac{\partial p}{\partial x_{i}}\right)+f_{i}
\end{gathered}
$$

where

$$
\tau_{i j}=\mu_{0}\left(\frac{\partial v_{i}}{\partial x_{j}}+\frac{\partial v_{j}}{\partial x_{i}}\right)
$$

In the above equations, $\rho$ is the fluid density, $v$ is the fluid velocity vector, $p$ is the fluid pressure, $f$ is the acceleration due to an external body force acting on the fluid, $\mu_{0}$ is the fluid viscosity, and $\tau_{i j}$ is the stress tensor. For viscous, continuum gas flows, the velocity field is subject to the no-slip boundary condition. The no-slip boundary condition imposes that the fluid adjacent to the wall has zero velocity relative to the wall. Further simplifying assumptions allow the system to be solved analytically for Couette and Poiseuille flows. Couette flow is a constant density, one-dimensional, fully-developed, steady flow where one wall boundary is moving relative to the other causing the viscous forces to drive the flow. Poiseuille flow is a constant density channel flow where the flow is driven by a pressure gradient or body force, and is one-dimensional, fully-developed, steady flow. Couette and Poiseuille flow conditions are used in the investigation because analytical continuum solutions are easy to obtain. In order to quantify the quality with which the analytical Navier-Stokes solutions capture the DSMC cases in the database, the non-dimensional $L_{2}$ error norm defined in (10) is used as a measure of the error.

$$
L_{2}=\frac{1}{\bar{v} N} \sqrt{\sum_{i=1}^{N}\left[\left(v_{\mathrm{mc}}\right)_{i}-\left(v_{\mathrm{ns}}\right)_{i}\right]^{2}} \quad \text { where } \bar{v}=\frac{1}{N} \sum_{i=1}^{N}\left(v_{\mathrm{mc}}\right)_{i}
$$

and $\left(v_{\mathrm{mc}}\right)_{i}$ and $\left(v_{\mathrm{ns}}\right)_{i}$ are the DSMC and Navier-Stokes velocity in cell $i$, respectively.

\subsection{Couette flow}

Both the no-slip boundary condition and the shear stress closure used in the continuum solution breakdown when the flow deviates from non-equilibrium. In Figure 1, the nonequilibrium DSMC solution (circles) for the Couette velocity profile demonstrates the violation in the no-slip boundary condition for Knudsen numbers ranging from 0.01 to 10 . All the Couette solutions shown are for a fixed duct height of $1 \mathrm{~mm}$ with the upper wall moving at $20 \mathrm{~m} / \mathrm{s}$ to the right relative to the stationary lower wall. The number of collisions between gas molecules near the wall decreases with increased rarefaction and the fluid deviates further from the conditions at the wall resulting in more slip. As the Knudsen number increases, so does the difference between the wall velocity and the adjacent fluid velocity, as shown in Figure 1. Maxwell derived from first principles the result that the velocity slip of the fluid 


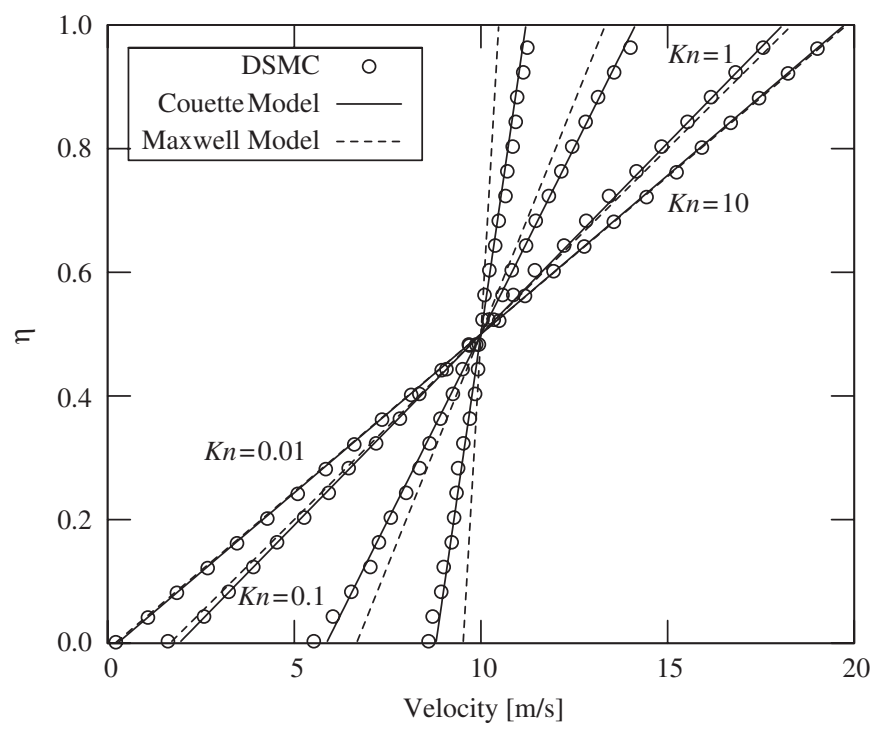

Figure 1. The non-equilibrium DSMC velocity profiles for Couette flow predict an increase in the slip velocity with Knudsen number. The linear Navier-Stokes solutions using the empirical model from this investigation are able to capture the DSMC profile within an $L_{2}$ error norm of $3 \%$.

at the wall for near continuum flows is proportional to the mean free path of the gas and the velocity gradient. However, Maxwell's model (dashed line in Figure 1.) over-predicts the slip velocity by at least $10 \%$ for $K n \geqslant 1$. Many researchers have added a slip coefficient $C_{\text {s }}$ to Maxwell's slip model (1) to match certain analytical, numerical, and experimental results [4-11]. The value is not always the same and generally depends on the flow type and the non-equilibrium results used; however, the consensus for near equilibrium flows is $C_{\mathrm{s}} \approx 1$.

The Navier-Stokes velocity profile for Couette flow is simply a straight line between the two wall velocities. Using the slip model, it is possible to judiciously choose the slip coefficient to match the straight line solution of the Navier-Stokes equations to the non-equilibrium solutions predicted by DSMC. In Figure 1, the Navier-Stokes solution (solid line) using the model slip coefficient developed in this investigation is shown to be in good agreement with the DSMC solution (circles) for Couette velocity profiles with Knudsen numbers ranging from 0.01 to 10. While the non-equilibrium DSMC solutions are not straight lines, the slight curvature they possess in the Knudsen layer near the wall makes the straight line approximation reasonable. The Navier-Stokes solution matches the DSMC Couette solutions within an $L_{2}$ error norm of $3 \%$ for Knudsen numbers ranging from 0.01 to 10 . This means that the right slip coefficient can yield accurate velocity profiles for non-equilibrium Couette flows. Even if the best slip coefficient is chosen to match the non-equilibrium velocity data, the continuum based solution cannot predict the shear stress. In Figure 2, predictions of the shear stress are presented for Couette flows ranging in Knudsen number from 0.01 to 10. As the Knudsen number increases, the amount the Navier-Stokes solution over-predicts the shear stress increases which indicates that the apparent fluid viscosity used in the shear stress closure is too high despite the slip correction. In this investigation, a similar viscosity correction to the one proposed by 


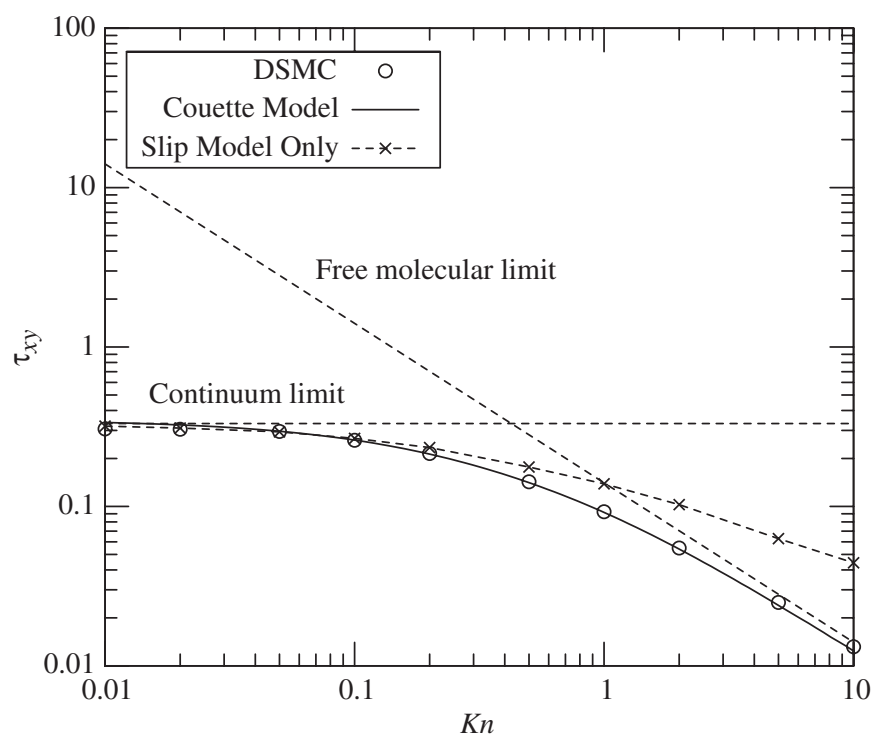

Figure 2. The error in the transport closure grows with increasing Knudsen number even if the best slip coefficient is used with the Navier-Stokes solution.

Karniadakis and Beskok is used [8]. The corrected viscosity $\mu^{\prime}$ is the apparent fluid viscosity needed to capture the non-equilibrium shear stress predicted by DSMC

$$
\mu^{\prime}=\mu_{0} C_{\mu}(K n)
$$

It is obtained by multiplying the standard fluid viscosity $\mu_{0}$ by an empirical correction $C_{\mu}$ that can depend on the Knudsen number and flow type. This correction $C_{\mu}$ is referred to in this work as the viscosity model coefficient.

\subsection{Poiseuille flow}

Poiseuille flow is more complex because both the velocity boundary condition and the shear stress closure affect the velocity profile. As a result, both the slip coefficient and viscosity model coefficient must be tuned to match the Navier-Stokes solution to the non-equilibrium solution. In Figure 3, the non-equilibrium DSMC solutions (circles) show an increase in slip velocity with Knudsen number similar to Couette flow. The non-equilibrium profiles are not exactly parabolic, but the parabolic solutions of the corrected Navier-Stokes equation (solid lines) are able to capture the non-equilibrium velocity profiles within a $7 \% L_{2}$ error norm, as shown in Figure 3. The wall shear stress of Poiseuille flow is independent of the shear stress closure, so the shear stress data is not considered in this investigation. It is demonstrated that with the slip and viscosity model coefficients developed in this investigation it is possible to stretch the Navier-Stokes solutions to match non-equilibrium Couette and Poiseuille flows with Knudsen numbers in the range of $0.01 \leqslant K n \leqslant 10$. 


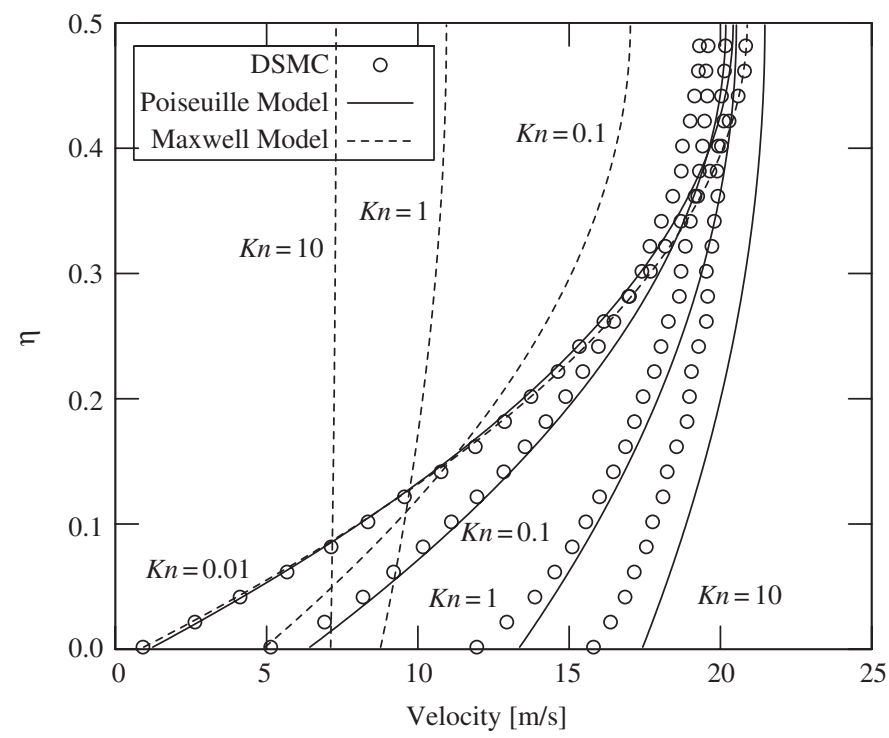

Figure 3. The non-equilibrium DSMC profiles for Poiseuille flow predict an increase in slip velocity with Knudsen number. The linear Navier-Stokes solutions using the empirical model from this investigation are able to capture the DSMC profile within an $L_{2}$ error norm of $7 \%$.

\section{INVESTIGATION METHOD}

It is possible to obtain accurate continuum-based Navier-Stokes solutions to non-equilibrium Couette and Poiseuille flows given appropriate slip and viscosity corrections. Unfortunately, these corrections require a pre-existing knowledge of the non-equilibrium solutions one wants to predict. Assuming one was given a database of non-equilibrium solutions, it would be possible to generate the slip and viscosity coefficients to yield the best possible Navier-Stokes solution for each flow in the database, which could then serve as a model for other flows. In this investigation, DSMC is used to provide the database of non-equilibrium Couette and Poiseuille solutions for argon and nitrogen gas flows with Knudsen numbers ranging from 0.01 to 10. The slip and viscosity coefficients for the Navier-Stokes solutions are found that yield the closest approximation to the DSMC solutions in a linear least-squares sense. The generated coefficients for each case in the database are separated into Couette and Poiseuille models in order to capture the Knudsen number dependence of the slip and viscosity coefficients. Finally, our Couette and Poiseuille models are used to predict non-equilibrium flows outside the database to evaluate their overall performance.

\subsection{DSMC procedures}

The database of non-equilibrium solutions used to generate our models consists of onedimensional argon and nitrogen Couette and Poiseuille flows. The DSMC solutions used for the database are obtained from a modified version of the one-dimensional code provided by Bird [1]. All flows in the database are low speed flows, the Poiseuille flows are driven to 
a maximum velocity of $20 \mathrm{~m} / \mathrm{s}$ and the Couette flows use a difference in wall velocities of $20 \mathrm{~m} / \mathrm{s}$. Knudsen numbers $0.01,0.02,0.05,0.1,0.2,0.5,1,2,5$, and 10 are simulated by adjusting the operating density of the working fluid, while keeping the geometry and computational domain constant. The walls are simulated with a temperature of $273 \mathrm{~K}$ and fully diffuse gas-surface interaction resulting in a TMAC of unity. The DSMC simulation is onedimensional which implies that the velocity distribution function is everywhere uniform along planes parallel to the walls. As a result, a pressure gradient cannot be used to drive the Poiseuille flow cases; instead, the accelerative body force $f$ is used. The driving force varies with Knudsen number, and is found through trial and error until the DSMC results appear to have a maximum velocity of $20 \mathrm{~m} / \mathrm{s}$. The collision dynamics are simulated using the variable soft sphere model with the collision parameters from Bird, and the rotational energy exchange for the nitrogen gas flows is simulated with the Larsen and Borgnakke model [1]. All DSMC simulations use 150 cells and 4500 simulated particles, except for the case at $K n=0.01$ Couette flow which uses 300 cells and 9000 simulated particles. The maximum cell length is less than one third of a mean free path. The time step is chosen so that a particle will cross a cell in an average of three time steps. The results are sampled for 20 million time steps with the typical statistical scatter in the velocity profile less than $1 \%$.

\subsection{Generating the best slip and viscosity model coefficients}

The Navier-Stokes equation can be solved analytically for most Couette and Poiseuille flows that are one-dimensional, fully developed, steady, isothermal and constant density. These are the same conditions found in the DSMC simulations. Using the empirical slip model (1) and viscosity correction (11) introduced earlier, the resulting Navier-Stokes solutions for the flow velocity and shear stress are

$$
\begin{aligned}
& u_{\mathrm{c}}(\eta)=U_{1}+\frac{U_{2}-U_{1}}{1+2 \Lambda}(\eta+\Lambda) \\
& u_{\mathrm{p}}(\eta)=-\frac{F^{\prime}}{2}\left(\eta^{2}-\eta-\Lambda\right) \\
& \left(\tau_{x y}\right)_{\mathrm{c}}=\frac{\mu^{\prime}}{1+2 \Lambda}\left(U_{2}-U_{1}\right) / h
\end{aligned}
$$

and

$$
\left(\tau_{x y}\right)_{\mathrm{p}}=-\rho f h(\eta-1 / 2)
$$

In the above equations, $u_{\mathrm{c}}$ and $u_{\mathrm{p}}$ are the Couette and Poiseuille velocity profiles, $\left(\tau_{x y}\right)_{\mathrm{c}}$ and $\left(\tau_{x y}\right)_{\mathrm{p}}$ are the Couette and Poiseuille flow shear stress, $U_{1}$ and $U_{2}$ are the lower and upper wall velocities for the Couette flow, $\eta=y / h$ is the wall normal coordinate non-dimensionalized by the channel height $h$, and $F^{\prime}=\rho f h^{2} / \mu^{\prime}$ is the body force term. The purpose of the empirical coefficients is to tune the continuum-based Navier-Stokes solutions to yield an approximation to the non-equilibrium DSMC results in the database. The Navier-Stokes velocity profile for Couette flow is simply a straight line with the following constraint that the velocity at the midpoint of the channel must be the average of the two wall velocities. Therefore, any line 
of the form in (16) is a valid Navier-Stokes solution for shear-driven flow

$$
u_{\mathrm{c}}(\eta)=G_{\mathrm{c}}\left(\eta-\frac{1}{2}\right)+\frac{1}{2}\left(U_{1}+U_{2}\right)
$$

where $G_{\mathrm{c}}$ is a free parameter that characterizes the family of solutions for different slip coefficients.

We can select the Navier-Stokes solution from this family that best fits the DSMC data by performing a linear least-squares fit of the DSMC velocity profile using

$$
G_{\mathrm{c}}=\frac{\left(S_{u y}\right)_{\mathrm{c}}}{\left(S_{y y}\right)_{\mathrm{c}}}
$$

where

$$
\left(S_{y y}\right)_{\mathrm{c}}=\sum_{i=1}^{N}\left(\eta_{i}-\frac{1}{2}\right)^{2}
$$

and

$$
\left(S_{u y}\right)_{\mathrm{c}}=\sum_{i=1}^{N}\left[\left(u_{i}\right)_{\mathrm{c}}-\frac{1}{2}\left(U_{1}+U_{2}\right)\right]\left(\eta_{i}-\frac{1}{2}\right)
$$

In the above equations, $\eta_{i}$ and $\left(u_{i}\right)_{\mathrm{c}}$ are the non-dimensional position and velocity, respectively, in the $i$ th DSMC cell, and $N$ is the total number of cells. Once the free parameter $G_{\mathrm{c}}$ is found for the best fitting solution, it can be related back to the slip coefficient to determine the slip boundary condition necessary to best capture the non-equilibrium flow

$$
C_{\mathrm{s}}(K n)=\frac{U_{2}-U_{1}-G_{\mathrm{c}}}{2\left(\left(2-\sigma_{v}\right) / \sigma_{v}\right) K n G_{\mathrm{c}}}
$$

As mentioned earlier, matching the velocity in Couette flow is only half the problem. In order to capture the correct shear stress, the viscosity model coefficient that yields the closest fit to the DSMC results is found and is related to the free parameter $G_{\mathrm{c}}$ found for the best slip boundary for the Navier-Stokes solution

$$
C_{\mu}(K n)=\frac{h}{\mu_{0} N G_{\mathrm{c}}} \sum_{i=1}^{N}\left(\tau_{x y}\right)_{i}
$$

where $\left(\tau_{x y}\right)_{i}$ is the shear stress in the $i$ th DSMC cell.

The Navier-Stokes solution for the Poiseuille flow velocity profile is a parabola with zero slope at the channel midpoint. This means that there are two free parameters $G_{\mathrm{p} 1}$ and $G_{\mathrm{p} 2}$ to characterize the family of valid Navier-Stokes solutions, with different boundary conditions and transport closures

$$
u_{\mathrm{p}}(\eta)=G_{\mathrm{p} 2}\left(\eta^{2}-\eta\right)+G_{\mathrm{p} 1}
$$

Similar to Couette flow, the values of $G_{\mathrm{p} 1}$ and $G_{\mathrm{p} 2}$ can be found that best match the nonequilibrium solution by performing a linear least-squares fit to each DSMC Poiseuille flow case in the database:

$$
\left[\begin{array}{c}
G_{\mathrm{p} 1} \\
G_{\mathrm{p} 2}
\end{array}\right]=\left[\begin{array}{cc}
N & \left(S_{y}\right)_{\mathrm{p}} \\
\left(S_{y}\right)_{\mathrm{p}} & \left(S_{y y}\right)_{\mathrm{p}}
\end{array}\right]^{-1}\left[\begin{array}{c}
\left(S_{u}\right)_{\mathrm{p}} \\
\left(S_{u y}\right)_{\mathrm{p}}
\end{array}\right]
$$


where

$$
\begin{aligned}
\left(S_{y}\right)_{\mathrm{p}} & =\sum_{i=1}^{N}\left(\eta_{i}^{2}-\eta_{i}\right) \\
\left(S_{y y}\right)_{\mathrm{p}} & =\sum_{i=1}^{N}\left(\eta_{i}^{2}-\eta_{i}\right)^{2} \\
\left(S_{u}\right)_{\mathrm{p}} & =\sum_{i=1}^{N}\left(u_{i}\right)_{\mathrm{p}}
\end{aligned}
$$

and

$$
\left(S_{u y}\right)_{\mathrm{p}}=\sum_{i=1}^{N}\left(u_{i}\right)_{\mathrm{p}}\left(\eta_{i}^{2}-\eta_{i}\right)
$$

Once the free parameters $G_{\mathrm{p} 1}$ and $G_{\mathrm{p} 2}$ are found, they uniquely determine the slip coefficient and viscosity model coefficient that will best capture the non-equilibrium velocity profile in a linear least-squares sense:

$$
C_{\mathrm{s}}(K n)=-\frac{G_{\mathrm{p} 1}}{G_{\mathrm{p} 2}\left(\left(2-\sigma_{v}\right) / \sigma_{v}\right) K n}
$$

and

$$
C_{\mu}(K n)=-\frac{\rho f h^{2}}{2 G_{\mathrm{p} 2} \mu_{0}}
$$

Using the database of DSMC cases as reference, it is possible to generate the best slip and viscosity model coefficients to match the Navier-Stokes solution to each non-equilibrium solution.

\subsection{Constructing the models}

The optimal slip and viscosity model coefficients are found using the linear least-squares method for each of the 40 DSMC cases in the database. In Figure 4, the optimal coefficients are plotted for argon gas (crosses) and nitrogen gas (squares) with separate results for Couette and Poiseuille flow. The optimal slip coefficient for Couette flow at a Knudsen number equal to 0.01 is between 1.8 and 1.9, which is higher than expected for the near continuum limit based on previously reported values ranging from 1.1 to $1.3[10,11]$. The optimal slip coefficient for Poiseuille flow and both viscosity model corrections are near unity at a Knudsen number equal to 0.01 , which is consistent with other models in the literature and the fluid viscosity in the continuum limit [8]. In all cases, the coefficients exhibit the trend of decreasing magnitude for transitional Knudsen numbers ranging from 0.1 to 10 . For Couette flow at a Knudsen number equal to 10 , the optimal slip coefficient decreased to 0.4 , while the result was much lower for Poiseuille flow which approached 0.1 at the same condition. The optimal viscosity model coefficients exhibited similar behaviour, decreasing to 0.3 and 0.05 for Couette and Poiseuille flows, respectively, at a Knudsen number equal to 10 . The variation in the optimal coefficients 

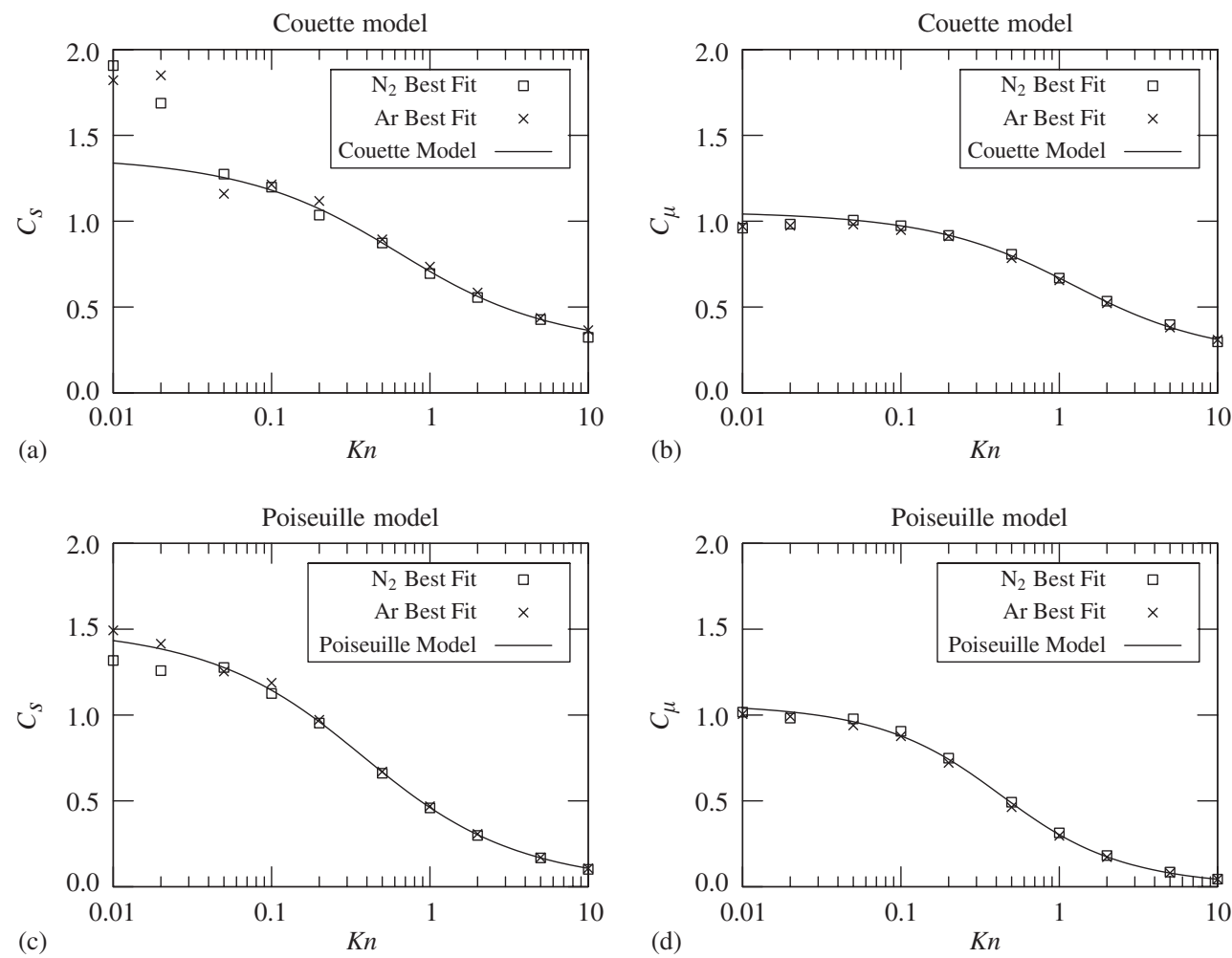

Figure 4 . The best slip $C_{\mathrm{s}}(K n)$ and viscosity $C_{\mu}(K n)$ coefficients are found that match the Navier-Stokes solution in a linear least-squares sense to the DSMC solution for each condition in the database: (a) Couette flow slip coefficient; (b) Couette flow viscosity model coefficient; (c) Poiseuille flow slip coefficient; and (d) Poiseuille flow viscosity model coefficient.

between gas species decreases as the Knudsen number increases. This results in a difference of less than a few per cent for Knudsen numbers greater than 0.1. Furthermore, at low Knudsen numbers at or below 0.2, the best coefficients found to match the DSMC data show the most deviation from the overall Knudsen number trend of the remaining data. This is attributed to the fact that the sensitivity of the slip coefficient is proportional to the Knudsen number. For example, a $100 \%$ change in $C_{\mathrm{s}}$ for Poiseuille flow at $K n=0.01$ results in only a $1 \%$ change in the velocity profile, while a $1 \%$ change in $C_{\mathrm{s}}$ at $K n=10$ results in a $10 \%$ change in the velocity profile. Therefore, any deviation at very low Knudsen numbers has little impact on the performance. The similarity of the molecular weights of argon and nitrogen combined with the isothermal cases used in the database probably account for the absence of a significant dependence on the gas species. The difference in the molecular weights results in a $16 \%$ difference in the most probable molecular velocity. Since all cases are nearly isothermal, there is not an appreciable difference in the activation of the energy modes between the monatomic argon and diatomic nitrogen.

The generation of a model to improve the Navier-Stokes solution in the non-continuum non-equilibrium regime would be a valuable tool for solving micro-scale gas flows rapidly. 
Table I. Parameters $a_{0}, a_{1}, a_{2}$, and $a_{3}$ selected in this investigation for the Couette and Poiseuille models having the form $C_{\mathrm{s}}=a_{0}+a_{1} \tan ^{-1}\left(a_{2} K n^{a_{3}}\right)$.

\begin{tabular}{lcccc}
\hline & Couette $C_{\mathrm{s}}$ & Couette $C_{\mu}$ & Poiseuille $C_{\mathrm{s}}$ & Poiseuille $C_{\mu}$ \\
\hline$a_{0}$ & 1.382 & 1.057 & 1.515 & 1.066 \\
$a_{1}$ & 0.717 & 0.547 & 0.962 & 0.679 \\
$a_{2}$ & -1.372 & -0.859 & -1.941 & -2.082 \\
$a_{3}$ & 0.677 & 0.745 & 0.680 & 0.866 \\
\hline
\end{tabular}

Empirical slip and viscosity models are created for both Couette and Poiseuille flows by fitting the functional form of the BPB slip model (2) to the optimal coefficients found in Figure 4. Once a suitable function is found, it provides an explicit evaluation of the coefficients over a large range of Knudsen numbers [10]. The resulting models are nonlinear so the least-squares method presented earlier cannot be applied directly to find the best fit. As a result, the free parameters that determine the nonlinear scaling in the Knudsen number, $a_{2}$, and $a_{3}$, are found using a direct search over a wide range of values, while the remaining parameters are found through a simple linear regression. The final values for the free parameters are chosen to yield the smallest error when recovering the optimal coefficients for the DSMC database. Both gas species are included in the Couette and Poiseuille models to see if the more general set of non-equilibrium solutions can retain accuracy similar to the BPB and KB models. The free parameters used for our Couette and Poiseuille models' slip and viscosity model coefficients, $a_{0}, a_{1}, a_{2}$, and $a_{3}$, are presented in Table I. In Figure 4, our models (solid lines) are shown to be in good agreement with the optimal coefficients for both argon and nitrogen gas at Knudsen numbers greater than 0.02. Results for Knudsen numbers less than 0.02 are excluded from our models because of the insensitivity of the coefficients within this range. It is important to note that the viscosity model coefficient represents the errors in the continuum solution for the Couette flow shear stress, and the Poiseuille flow mass flux if no shear stress closure correction is used. In order to achieve a $20 \%$ accuracy in those flow quantities, the viscosity model coefficient must be used when it is less than 0.8 . The viscosity model coefficient drops below 0.8 for Couette flows with Knudsen numbers of 0.5 and greater, and for Poiseuille flows with Knudsen numbers greater than 0.1 .

\subsection{Predictive cases}

Great care is taken to ensure the empirical Couette and Poiseuille models developed in this investigation for the slip and viscosity model coefficients capture the non-equilibrium flows in the DSMC database. However, the accuracy of the models for cases within the database is not a measure of the models' applicability, only of the data fit correlation. Our models are not predicting the non-equilibrium flows from the database, they are just carefully tuned to reproduce them. Since our models are purely empirical and the continuum based solutions that use them break down as the flow deviates from equilibrium, they should be suspect when predicting flows outside the database at large Knudsen numbers. In order to assess the actual predictive power of our Couette and Poiseuille models, five types of test cases outside the DSMC database are selected to illustrate different non-equilibrium challenges. The models developed in this investigation are used to predict these flows with the results 
compared to DSMC results. The first cases involve interpolation and extrapolation of the database for both Couette and Poiseuille argon flows at Knudsen numbers of 0.7 and 20 . Second, a combination Couette and Poiseuille flow is simulated for nitrogen gas at $K n=1$. Third, the tangential momentum accommodating coefficient (TMAC) is changed from unity to 0.8 and 0.5 for both Couette and Poiseuille argon flows. Fourth, helium gas is used as the working fluid for Couette and Poiseuille flows at $K n=1$. Helium has a molecular weight about one tenth of that of argon, so the resulting most probable molecular velocity is three times that of argon. Finally, a body force driven flow with uniform suction and injection normal to the walls is simulated at $K n=1$. While the solution is still one-dimensional, it is the only flow in this investigation that has a non-zero convective acceleration. Most multidimensional flows, or flows with complex geometry have a non-zero convective acceleration, so the ability of our Poiseuille model to capture the physics change in this flow is an indication of the applicability of our models toward more complex flows. The analytical solution to the body force driven flow with a uniform suction and injection velocity $V_{0}$ at the walls is given in following equations [12]:

$$
u_{\chi}=\frac{F^{\prime}}{\operatorname{Re}}\left[D\left(\mathrm{e}^{\eta R e}+\Lambda R e-1\right)+\eta+\Lambda\right]
$$

where

$$
D=\frac{1+2 \Lambda}{1-\Lambda R e-(1+\Lambda R e) \mathrm{e}^{R e}}
$$

$R e=\rho V_{0} h / \mu^{\prime}$ is the non-dimensional Reynolds number based on the cross flow velocity $V_{0}$ and the corrected fluid viscosity $\mu^{\prime}$, and $F^{\prime}$ is the same force term as the Poiseuille solution (13).

\section{DATABASE ACCURACY OF THE MODELS}

The Couette and Poiseuille models developed in this investigation are designed to reproduce the non-equilibrium DSMC simulations in the database. Since our models are derived directly from the non-equilibrium solutions in the DSMC database, their ability to recreate the DSMC results in the database is a measure of the models' correlation to known data and not necessarily its predictive power. However, the models' correlation to the DSMC results is important in assessing the models' ability to interpolate between the flow conditions found in the database. The performance of our models is compared to the results using the best slip and viscosity model coefficients found at each flow condition which is the lower bound for the $L_{2}$ error norm of the models. In addition, the performance of the models developed in this investigation is compared to the BPB model for Couette flow and the KB model for Poiseuille flow. Since the Couette and Poiseuille models developed in this investigation do not include any information about the flow geometry or type, both are tested on each flow type to assess their sensitivity.

The ability of the Couette model developed in this investigation to simulate the Couette flows in the DSMC database is evaluated based on the $L_{2}$ error norm in the velocity profile, the normalized error in the slip velocity at the wall, and the error in the normalized shear stress. The results are similar for both argon and nitrogen gases, so only the results for nitrogen are presented and discussed. In Figure 5, the normalized error between the model corrected 

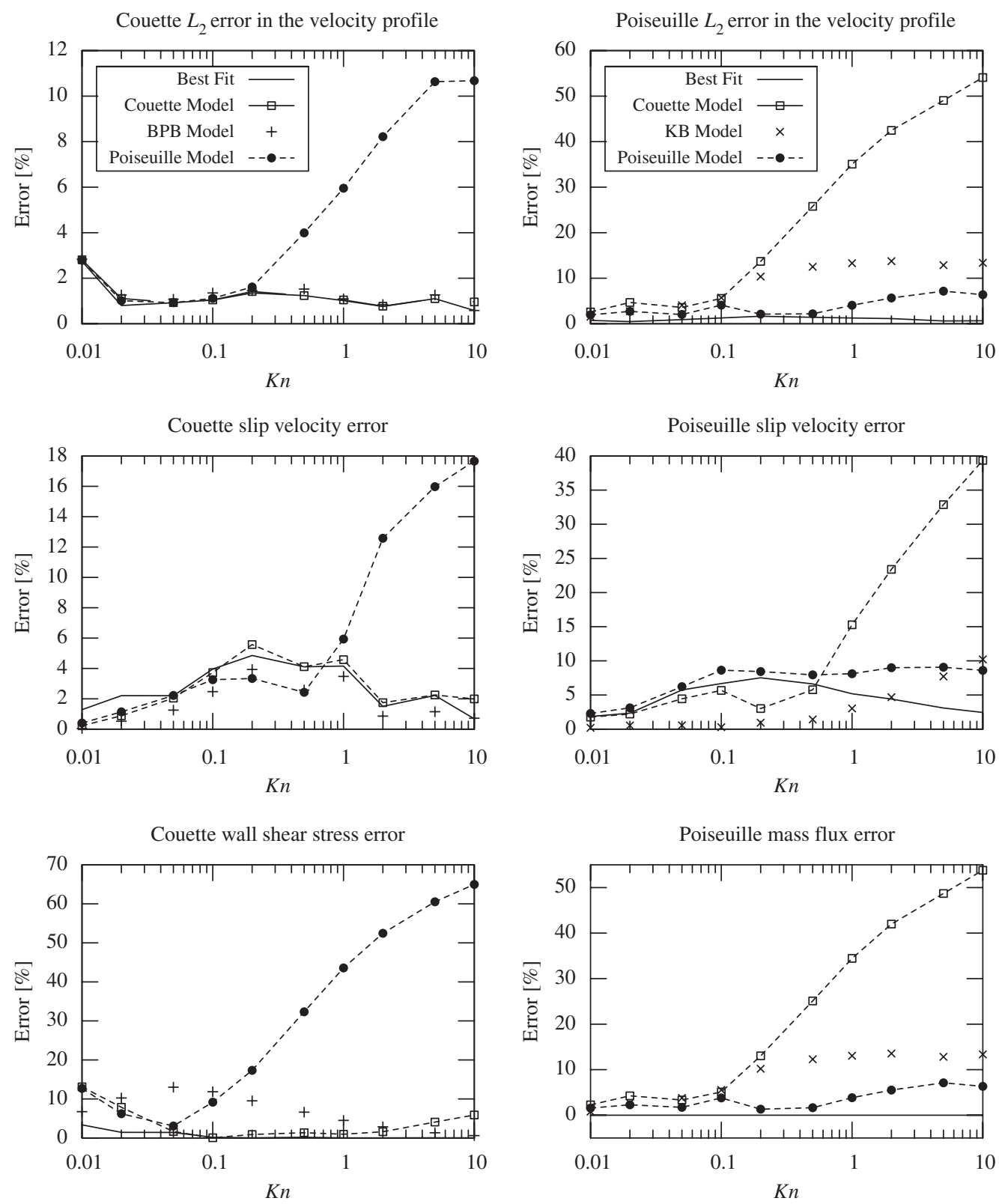

Figure 5. Error in the Navier-Stokes solution using various slip and viscosity models for Couette flow (left) and Poiseuille flow (right).

Navier-Stokes solution and DSMC is presented for Couette flows in the database using: the optimum Navier-Stokes solution (solid line), our Couette model (dashed/squares), the BPB model (crosses), and our Poiseuille model developed in this investigation (dashed/circles). 
The $L_{2}$ error norm of the Couette velocity profiles produced by our Couette model and the BPB model are within 3\% for all Knudsen numbers. Also, all models perform equally well for Knudsen numbers less than or equal to 0.2 . It is only above this level when our Poiseuille model diverges. Even at its worst, our Poiseuille model is still within an $L_{2}$ error norm in the velocity profile of $11 \%$. The absence of a viscosity dependence in the velocity for the continuum Couette solution prevents any errors in the shear stress closure from affecting the velocity profile. As a result, non-equilibrium Couette flow cases can be especially forgiving when modelled with continuum solutions. The accuracy is similar for the error in the slip velocity of the Couette flows in the database as shown in Figure 5. Again, our Couette model and the BPB model do well and capture the non-equilibrium slip velocity within $6 \%$ for all Knudsen numbers. The maximum error in the slip velocity simulated by the models occurs at $K n=0.2$ because this is when the DSMC solution has the greatest curvature near the walls. Our Couette model and the BPB model capture the shear stress within $12 \%$ for all Knudsen numbers. The error at the Knudsen numbers below 0.05 can be attributed in part to the statistical scatter still present in the DSMC simulation of the shear stress. Capturing the shear stress at high Knudsen numbers is much more sensitive than the velocity profile, as our Poiseuille model produces errors of $25 \%$. It is important to note that all models perform equally well for Knudsen numbers below 0.2 , which is consistent with the asymptotic behaviour of any slip model of the form (1) as $K n \rightarrow 0$. It is only as the Knudsen number increases beyond 0.2 that the performance becomes very sensitive to the flow type and the database used to construct the model.

The ability of our Poiseuille model to simulate the Poiseuille flows in the DSMC database is evaluated based on the $L_{2}$ error norm in the velocity profile, the normalized error in the slip velocity at the wall, and the error in the normalized mass flux. The error in the mass flux is an important engineering design factor for Poiseuille flows. For this reason, it is presented in lieu of the wall shear stress which only depends on the density, body force and duct height (15); and is independent of Knudsen number. In Figure 5, the normalized errors for the velocity profile, slip velocity, and mass flux are presented for the optimum Navier-Stokes solution (solid line), our Poiseuille model (dashed/circles), the KB model (crosses), and our Couette model (dashed/squares). The $L_{2}$ error norm in the velocity profile for our Poiseuille model developed in the investigation is less than $7 \%$ for all Knudsen numbers. The KB model captures the non-equilibrium DSMC velocity profiles within an $L_{2}$ error norm of $13 \%$ for all Knudsen numbers. Our Couette model diverges from the rest at Knudsen numbers greater than 0.1 , and is plagued with a large error greater than $50 \%$ at $K n=10$. The $\mathrm{KB}$ model is more accurate in capturing the slip velocity than the rest of the models, but our Poiseuille model still remains within $8 \%$ for the entire Knudsen regime. The error in the mass flux is bounded from above by the $L_{2}$ error norm so the trends are very similar, as Figure 5 demonstrates. Our Poiseuille model developed in this investigation accurately captures the mass flux within $7 \%$ for the entire Knudsen regime while the KB model is accurate within 13\%. All models capture the Poiseuille flow for Knudsen numbers less than or equal to 0.1, again preserving the near-continuum accuracy inherent in every slip model of the form of (1). The discrepancy between our models and the BPB and KB models is not a measure of the models' quality; rather, it is a reflection of the sensitivity of the models to the database upon which they are based. It is only as the flows become more rarefied that the accuracy of the models becomes sensitive to the database used to construct them. Overall, the Couette and Poiseuille models developed in this investigation have demonstrated that they can capture the non-equilibrium 
solutions in the DSMC database throughout the Knudsen number regime to a similar accuracy of other unified models found in the literature.

\section{MODEL PREDICTION PERFORMANCE}

The investigation has demonstrated that it is possible to construct models for the slip and viscosity coefficients so that the corrected Navier-Stokes solutions accurately capture the nonequilibrium solutions in the DSMC database. However, this is only a measure of the quality of the data fit the models are able to achieve, and is not a testament to their use as a predictive design tool. In order to understand the ability of a model constructed from a database of nonequilibrium solutions to predict other flows outside the database, the following five cases are tested: interpolation and extrapolation of the DSMC cases, combined Couette and Poiseuille flow, wall surfaces with partial momentum accommodation, helium gas flows, and channel flows with uniform suction and injection. In each case, only the Couette and Poiseuille models developed in this investigation are compared to the DSMC results. In Figures 1 and 3, the velocity profile of the Navier-Stokes solution using our models (solid line) is presented with the non-equilibrium solution from the DSMC database for nitrogen Couette and Poiseuille flows at $K n=0.01,0.1,1$, and 10. As a reference for the quality of the predictions, the results in Figure 1 demonstrate the ability of our Couette model to capture the velocity profile of the DSMC Couette solution within an $L_{2}$ error norm of $1 \%$, an over-prediction of the slip velocity by $3 \%$, and a shear stress error of $4 \%$. Similarly for Poiseuille flow in Figure 3, our Poiseuille model is able to capture the velocity profile of the DSMC solution within an $L_{2}$ error norm of $3 \%$, the slip velocity is $3 \%$ larger, and the mass flux is $3 \%$ larger.

\subsection{Interpolation and extrapolation}

Couette and Poiseuille argon gas flows are predicted using the models at $K n=0.7$ and 20, which is an interpolation and extrapolation of the cases used in the databases. The models predict all measurable error quantities within the baseline $K n=1$ accuracy. This indicates that an empirical model can serve as a tool to evaluate different operating densities of the same geometry over a wide range of Knudsen numbers, if there are enough non-equilibrium solutions available to construct the model. The number of non-equilibrium cases required in the database depends on the fidelity hoped to be achieved by the model and the complexity of the Knudsen number dependence of the system. However, it is not conclusive that the model affords any advantage over interpolating the slip and viscosity coefficients obtained directly from the least-squares analysis.

\subsection{Combination of Couette and Poiseuille flow}

The simplified Navier-Stokes equations for the Couette and Poiseuille flows considered in this investigation each reduce to a single linear differential equation for the velocity profile. Therefore, in the continuum limit, a flow that is a combination of Couette and Poiseuille flows can be solved as the superposition of a Couette solution and a Poiseuille solution. However, the Boltzmann equation, which is valid for flows ranging from continuum to free molecular, has a nonlinear collision term which prevents the linear superposition of the two flows in the transition regime. If the velocity distribution functions within the flow are still close to 


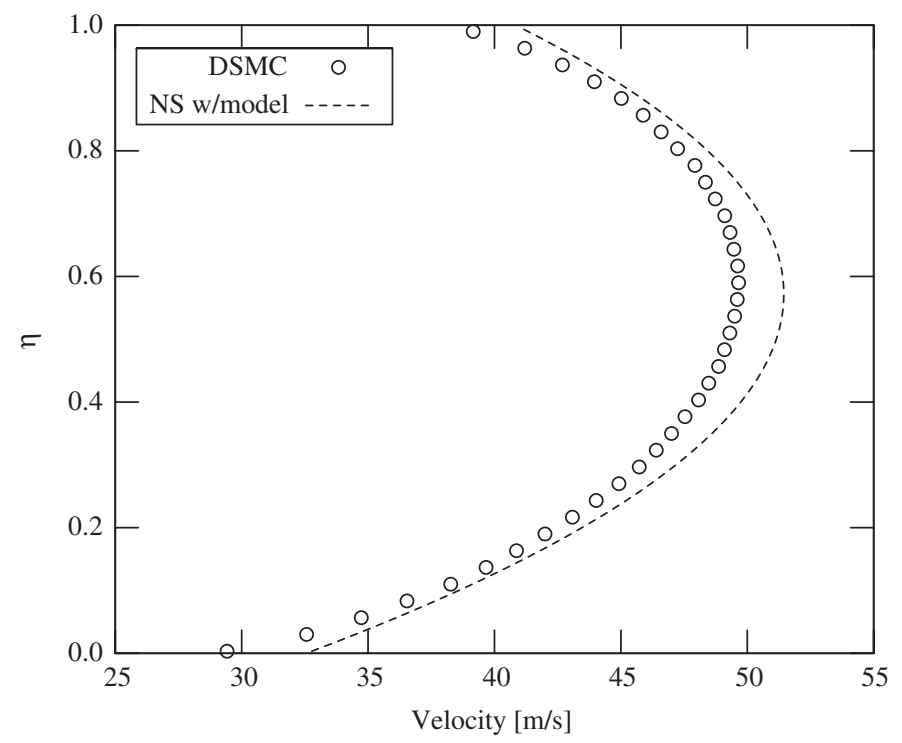

Figure 6. Comparison of the velocity profiles for combined Couette and Poiseuille flow at $K n=1$ between the DSMC solution and the Navier-Stokes solution using the models developed in this investigation.

equilibrium, then the error due to the non-linearity is small. In order to evaluate the effect of the non-linearity, a combination Couette and Poiseuille nitrogen flow at $K n=1$ is tested. The Navier-Stokes result is obtained by decomposing the flow into a Couette and Poiseuille contribution, solving each separately with the Couette and Poiseuille models developed in this investigation, and then adding the two solutions together under the principle of superposition. In Figure 6, the DSMC solution (circles) and the Navier-Stokes solution (solid line) using our models are presented for the combined Couette and Poiseuille velocity profile at $K n=1$. The lower wall is fixed while the upper wall moves at $20 \mathrm{~m} / \mathrm{s}$ in the direction of the driving force. The driving force combined with the moving wall boundary yield a maximum velocity of about $50 \mathrm{~m} / \mathrm{s}$ in the DSMC solution. The Navier-Stokes solution over-predicts the entire DSMC profile resulting in an $L_{2}$ error and mass flux error of $3 \%$, which is the same as the reference Poiseuille case at $K n=1$. Moreover, the shear stress is less than the reference Couette flow case and within the DSMC scatter. In Figure 6, the largest error in the Navier-Stokes prediction of the velocity profile occurs near the wall where the slip velocity is $7 \%$ larger than the DSMC result. This slip velocity error is about twice the error magnitude found in the reference Poiseuille case at $K n=1$. Overall, the effect of any non-equilibrium non-linearity appears small, and the decomposition of two flow types is appropriate in this case.

\subsection{Tangential momentum accommodation coefficient}

In order to determine the effect the TMAC has on the models' performance, argon gas Couette and Poiseuille flows are simulated for a TMAC equal to 0.8 and 0.5 at $K n=1$. For the Couette flows, the TMAC has no effect on the accuracy of the velocity profile, with the Couette model 

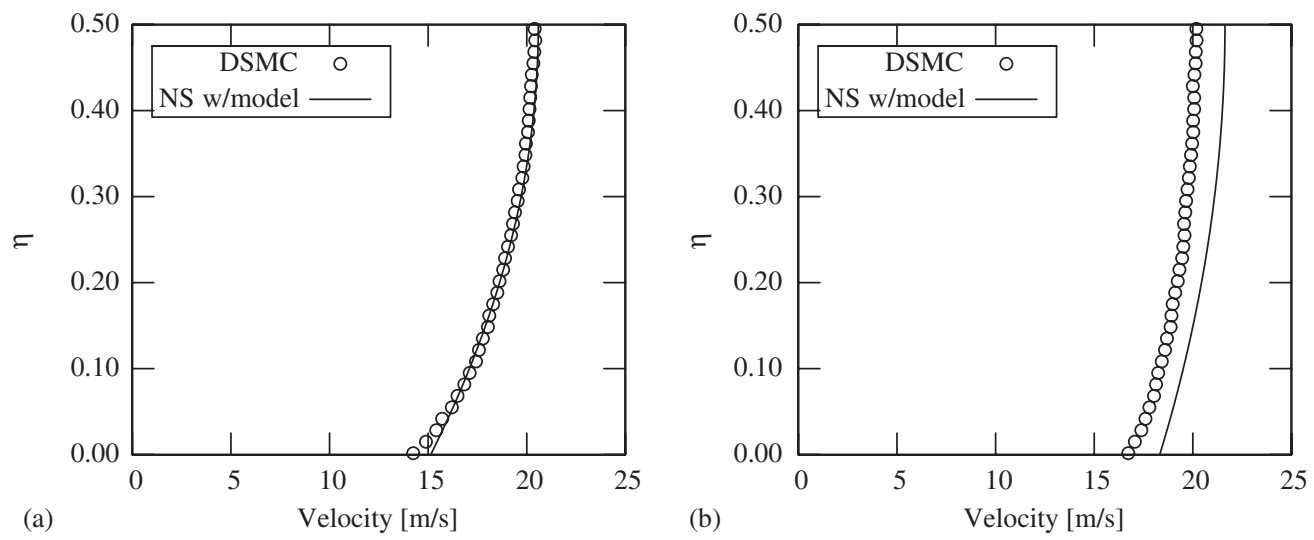

Figure 7. Comparison of the Navier-Stokes solution using the models from this investigation to the DSMC solution for Poiseuille flows at $K n=1$ : (a) TMAC $=0.8$; and (b) $\mathrm{TMAC}=0.5$.

predicting the profiles to the same accuracy as the baseline. However, the shear stress error doubled to $9 \%$ for a TMAC $=0.8$ and tripled to $13 \%$ for a TMAC $=0.5$. In Figure 7 , the DSMC solution (circles) and the Navier-Stokes solution (solid line) using our Poiseuille model are presented for the Poiseuille velocity profiles at $K n=1$, with the TMAC equal to 0.8 and 0.5 . For a TMAC $=0.8$, the difference between the velocity profiles predicted by DSMC and the Navier-Stokes equation is smaller than the reference Poiseuille case at $K n=1$, resulting in an $L_{2}$ error norm of $1 \%$. However, as shown in Figure 7, the Navier-Stokes prediction worsens when the TMAC equals 0.5. In this case, the Navier-Stokes solution with our Poiseuille model under-predicts the velocity across the entire channel with an error double that found in the reference Poiseuille case at $K n=1$. For a TMAC $=0.5$, the $L_{2}$ error in the velocity profile and the error in the mass flux is $7 \%$, and the slip velocity error is $8 \%$. The deviation from equilibrium is intricately coupled with the range of direct influence of the wall on the gas molecules of the flow. As the Knudsen number increases, so does the probability of finding a molecule whose last collision was with the wall. It is reasonable to assume that the TMAC will be a sensitive factor at higher Knudsen numbers. Therefore, care should be exercised when using an empirical model to predict a flow with a TMAC value different from that used in the database to construct the model.

\subsection{Helium gas flows}

The molecular weight of helium is one tenth that of argon, which means the most probable molecular speed of helium is over three times faster than argon. While there is no appreciable difference between the nitrogen and argon gas flows used to construct the Couette and Poiseuille models, their most probable molecular speeds are within $16 \%$. The large difference in molecular speeds between the helium cases and the models' database could affect the accuracy of the models' prediction. In order to evaluate this large change in molecular speeds, helium Couette and Poiseuille flows are simulated at $K n=1$. The helium gas is found to have no effect on the ability of our Couette model to predict Couette flow, all the measured errors are within the model accuracy for the baseline case. In Figure 8, the DSMC solution (circles) 


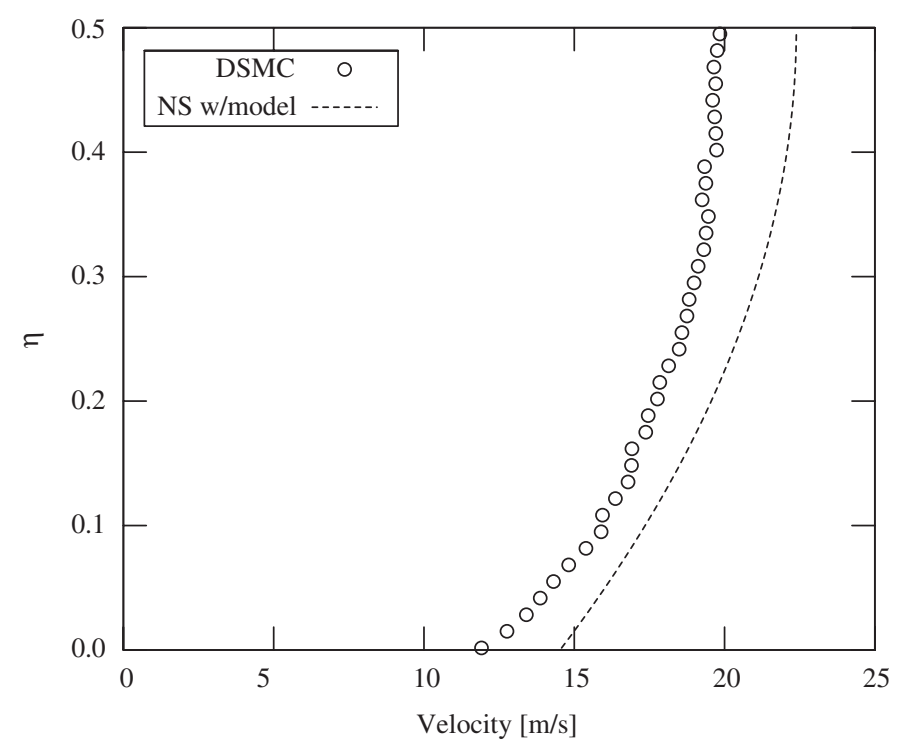

Figure 8. Comparison of the velocity profiles at $K n=1$ for helium gas Poiseuille flow between the DSMC solution and the Navier-Stokes solution using the models developed in this investigation.

and the Navier-Stokes solution (solid line) using our Poiseuille model are presented for the Poiseuille velocity profile of helium gas at $K n=1$. The increase in the random or thermal speed due to the lighter helium gas introduces more statistical scatter in the DSMC solution than the argon and nitrogen cases. The increased scatter in the velocity profile is illustrated in Figure 8, but the overall scatter is still less than 3\% across the channel. For the helium Poiseuille flow, the Navier-Stokes solution over-predicts the DSMC velocity profile throughout the channel resulting in a $12 \%$ higher mass flux than the DSMC solution. Furthermore, the errors in the $L_{2}$ norm of the velocity profile and slip velocity are 12 and $15 \%$, which is four to five times larger than the reference Poiseuille case at $K n=1$. The absence of error in the Navier-Stokes prediction for Couette flow is due to the viscosity independence of the velocity profile and further illustrates the model performance between flow types.

\subsection{Body force driven flow with uniform rates of suction and injection}

In order to test a one-dimensional flow with a non-zero convective acceleration, the boundary conditions for a body force driven flow are changed to include a uniform fluid injection at the lower wall and a uniform suction at the upper wall. In Figure 9, the DSMC solution (circles) and the Navier-Stokes solution (solid line) using our Poiseuille model are presented for the velocity profile of a body force driven flow with uniform rates of suction and injection for argon gas at $K n=1$. The body force is chosen to drive the flow at a maximum velocity of $20 \mathrm{~m} / \mathrm{s}$, while the injection and suction rates maintain a constant $20 \mathrm{~m} / \mathrm{s}$ cross flow. The presence of cross flow in the solution skews the normally symmetric Poiseuille velocity profile in the direction of the cross flow. This asymmetry in the DSMC solution creates a $10 \%$ difference between the slip velocity at the upper and lower wall boundaries, and shifts the 


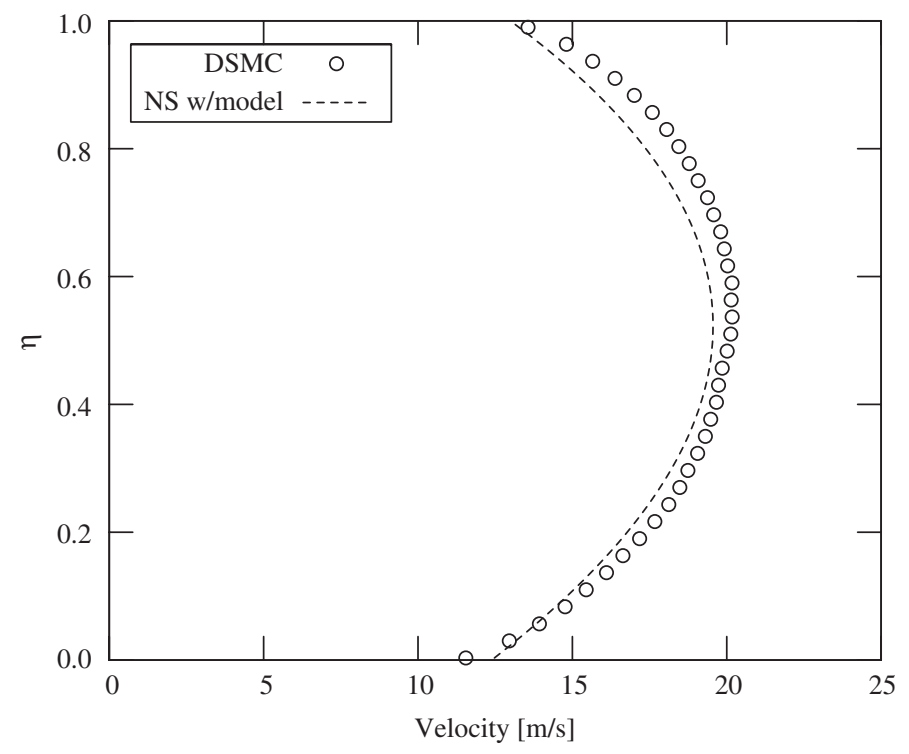

Figure 9. Comparison of the driven flow velocity profile with uniform suction and injection between the Navier-Stokes solution using the models developed in this investigation and the DSMC solution at the flow condition $K n=1$.

location of the maximum velocity from the centre by $8 \%$ of the channel width. The asymmetry in the Navier-Stokes solution due to the cross flow is not as pronounced as in the DSMC solution. As illustrated in Figure 9, the difference between the upper and lower slip velocities is less than $3 \%$ in the Navier-Stokes solution, while the location of the maximum velocity has shifted only $2 \%$ of the channel width. Furthermore, the difference in the velocity gradient at the walls between the DSMC and Navier-Stokes solutions creates an error in the wall shear stress of $8 \%$. The Navier-Stokes solution using our Poiseuille model is able to predict the mass flux within $3 \%$ of the DSMC solution, in spite of missing the key changes in the shape of the velocity profile due to the cross flow. The Poiseuille model does not appear to follow the change of physics for the non-zero convective acceleration. Thus, it is not recommended to use the Poiseuille model developed in this investigation for complex flows when the Knudsen number is larger than 0.1 .

\section{CONCLUSIONS}

There were two goals in this investigation. The first was to construct Couette and Poiseuille models based on empirical corrections in order for the Navier-Stokes solution to match a wide range of known non-equilibrium flows. The second was to evaluate their effectiveness as a predictive design tool. A database of non-equilibrium solutions was first simulated with DSMC for Couette and Poiseuille flows of argon and nitrogen, for Knudsen numbers ranging from 0.01 to 10 . Then the optimum slip and viscosity model coefficients for the Navier- 
Stokes solution were found for each flow condition so that the velocity profile and shear stress matched the DSMC data in a linear least-squares sense. Next, models were constructed for each flow type in order to capture the Knudsen number dependence of the slip and viscosity model coefficients. The Couette and Poiseuille models developed in this investigation demonstrated their ability to capture all the non-equilibrium results in the DSMC database for Couette flows with an $L_{2}$ error norm in the velocity profile of $3 \%$ and a shear stress error of $12 \%$. Similarly for Poiseuille flows, the Poiseuille model captured the results in the DSMC database with all velocity and mass flux errors within 7\%. The performance of the Couette and Poiseuille models developed here is similar to other unified models proposed by Beskok and Karniadakis, and Bahukudumbi, Park, and Beskok. All models, even those purposely used on flows that were not their intended design, were accurate for near equilibrium conditions at Knudsen numbers less than or equal to 0.1. Above this Knudsen number, the correction to the viscosity model coefficient indicates that the error in the shear stress closure is at least $10 \%$ for Couette flows and $20 \%$ for Poiseuille flows. The models' performance capturing the DSMC database was very sensitive in the transition and free molecular regimes. Generally, as the Knudsen number increases, so does the error using any model that was not explicitly constructed from the database used in the comparison. The Couette and Poiseuille models developed in this investigation were able to predict flows that are an interpolation or extrapolation of the DSMC database to a similar accuracy as the database cases themselves. In addition, a combination of both models was able to predict a combined Couette and Poiseuille flow in the transition regime. The Couette model was successful in predicting the velocity of all the cases because the Navier-Stokes solution is independent of any errors in the shear stress closure due to non-equilibrium. However, the Poiseuille model was not as successful in predicting flows with partial wall accommodation, helium gas, and non-zero convective acceleration terms. The models developed in this investigation are empirical corrections to a continuum solution that has little physical accuracy in the transition and free molecular regimes, and the errors found when pushing the models outside the database in these regimes are expected.

\section{ACKNOWLEDGEMENTS}

The first author gratefully acknowledges financial support of the Department of Energy through a Computational Science Graduate Fellowship administered by the Krell Institute and also would like to thank Sandia National Laboratories, in particular, the Engineering Sciences Center (dept. 9113), for their support of the investigation for this paper.

\section{REFERENCES}

1. Bird GA. Molecular Gas Dynamics and the Direct Simulation of Gas Flows. Clarendon: Oxford, 1994.

2. Milikan RA. Coefficients of slip in gases and the law of reflection of molecules from the surfaces of solids and liquids. The Physical Review 1923; 21(3):217-238.

3. Kennard EH. Kinetic Theory of Gases. McGraw-Hill: New York, 1938.

4. Karniadakis G, Beskok A. Micro Flows. Springer: New York, 2002.

5. Deissler RG. An analysis of second-order slip flow and temperature-jump boundary conditions for rarefied gases. International Journal of Heat and Mass Transfer 1964; 7:681-694.

6. Cercignani C. Mathematical Methods in Kinetic Theory. Plenum Press: New York, 1969.

7. Kogan MN. In Rarefied Gas Dynamics, Trilling L (ed.). Plenum Press: New York, 1969 (in Russian).

8. Beskok A, Karniadakis G. A model for flows in channels, pipes, and ducts at micro and nano scales. Journal of Microscale Thermophysics Engineering 1999; 3:43-77. 
9. Bahukudumbi P, Beskok A. A phenomenological lubrication model for the entire Knudsen regime. Journal of Micromechanics and Microengineering 2003; 13:873-884.

10. Bahukudumbi P, Park JH, Beskok A. A unified engineering model for steady and quasi-steady shear driven gas microflows. Microscale Thermophysical Engineering 2003; 7(4):291-315.

11. Pan LS, Liu GR, Lam KY. Determination of slip coefficient for rarefied gas flows using direct simulation Monte Carlo. Journal of Micromechanics and Microengineering 1999; 9(1):89-96.

12. White FM. Viscous Fluid Flow. McGraw-Hill: New York, 1991. 